\title{
THE UBVRI PHOTOMETRIC AND POLARIMETRIC OBSERVATIONS OF THE T TAU STAR HDE 283572
}

\author{
N. I. SHAKHOVSKAYA \\ Crimean Astrophysical Obsevatory \\ Nauchny \\ Crimea 334413 \\ USSR
}

ABSTRACT.The preliminary analysis of UBVRI photometric and polarimetric observations of the naked $\mathrm{T}$ Tau star HDE 283572 indicates that:

(i)there is intrinsic and variable component of polarisation;

(ii)the distribution and the area of the active regions on stellar surface are variable on time scale of a year.

\section{Introduction}

HDE 283572 is ninth-magnitude G-star south of RY Tau. As suggested by Walter et. al.(1987), HDE 283572 is a good example of a "naked T Tauri" (NTT) stars,i.e.,stars indentical to the low mass $\mathrm{T}$ Tauri stars save for the lack of significant circumstellar envelope. It was proposed, that the periodic ( $\approx 1.5$ ) modulation of magnitude in $\mathrm{V}\left(\approx 0^{\mathrm{m}} .2\right)$ is caused by asymmetric distribution of the active regions on the surface. This star may be very useful for studies of how the stellar (not circumstellar) activity levels behave in very young convective stars. Presented here are the preliminary results of my photometric and polarimetric observations.

\section{Observations and results}

The obsevations were made with the $125 \mathrm{~cm}$ telescope of Crimean Astrophysical Observatory using the simultaneous five color (UBVRI) photometer-polarimeter of Helsinki University.

\subsection{PHOTOMETRY}

In Fig. $1 \mathrm{~V}$ magnitudes folded on the ephemeris:J.D. $=2445600.173+1.548 \mathrm{E}$ by Walter et al. (1987) for the different seasons are given. The observations in 1983 were taking from Walter et al (1987). As follows from Fig 1, Walter's period does not contradict to my observations in the individual seasons. There are, however, a phase shift between different seasons and I could not improve period like that to fit all the observations. The amplitudes , maximal and minimal magnitudes of the light curve are variable. 


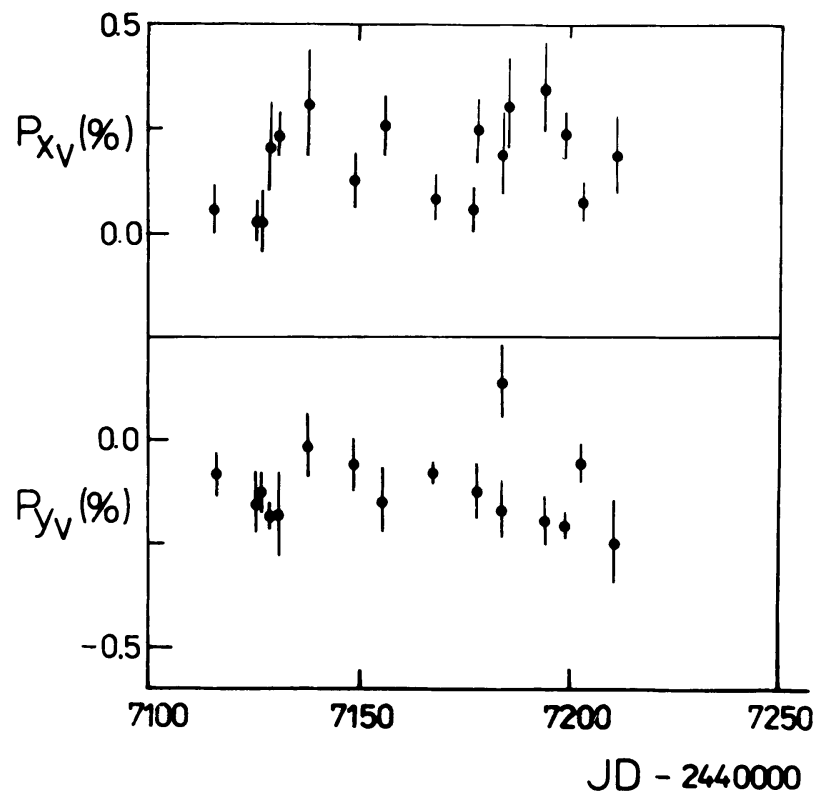

Figure 2. The components of polarization vector $\operatorname{Px}(\%)$ and $P y(\%)$ in $V$ band in 1987-88, plotted as function of Julian Days.

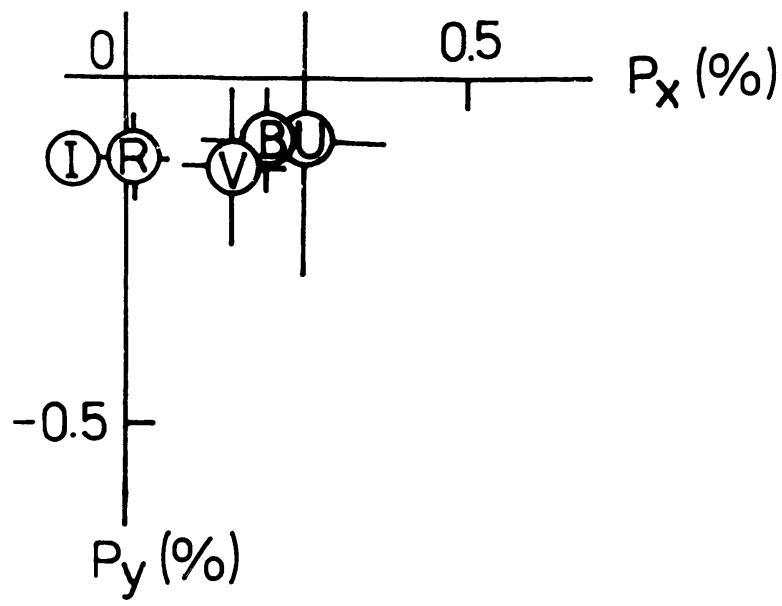

Figure 3. The polar diagram $(\mathrm{Px}, \mathrm{Py})(\%)$ for averaged data in UBVRI. 
The variability of the light curves and the phase shift could be explained by the change of the distributions and the area of the active regions on the stellar surface on the time scale of a year.

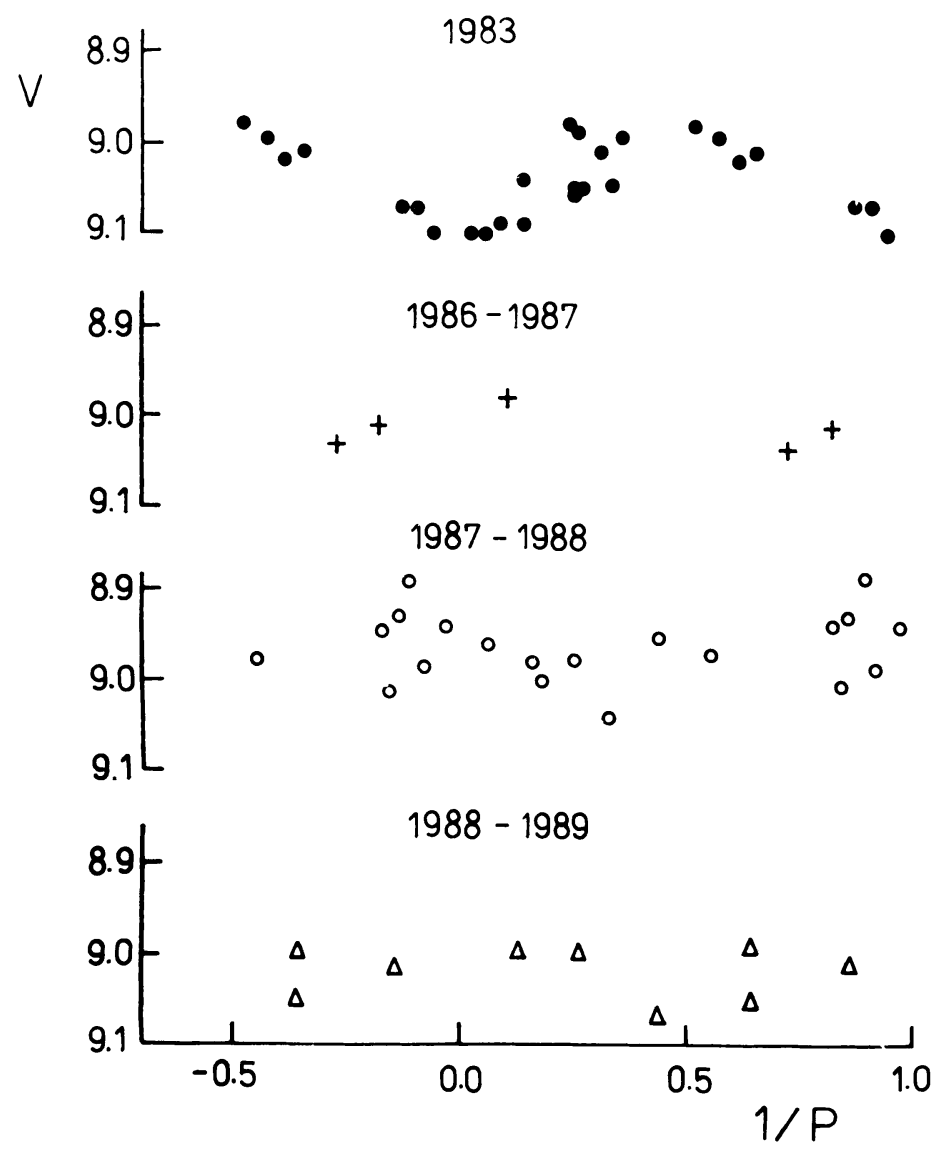

Figure 1. The light curves in $\mathrm{V}$ band on the ephemeris by walter et al.

There are strong linear correlations between magnitudes in the different bands, so: $\Delta U / \Delta V=1.17 \pm 0.10 ; \Delta B / \Delta V=1.10 \pm 0.06 ; \Delta R / \Delta V=0.82 \pm 0.04$; $\Delta I / \Delta V=0.73 \pm 0.03$. Thus, the brighter the star the bluer it is.

\subsection{POLARIMETRY}

The polarization in the $R$ and $I$ bands remained constant. But in the UBV the variation of polarization with amplitude $0.5 \%$ was observed. As an example in Fig. 2 the parameters Px and Py in $V$ band in 1987-88 are given $(P x=P \cos 2 \vartheta$ and $P y=P \sin 2 \vartheta$ are the components of the polarization vector $P$ in the $(F, 2 \vartheta) \mathrm{plane})$. But I could not find any correlation neither 
between Px,Py in different bands, nor between the polarization and photometric variability.

In Fig. 3 the Px and Py for UBVRI bands averaged for all data are given. As follows from Fig.3, there is strong rotation of the position angle $\vartheta$ as a function of the wavelength, from $\vartheta_{U}=150^{\circ}-190^{\circ}$ to $\vartheta_{I}=110^{\circ}$ $120^{\circ}$.

The variability of the polarization and wavelength dependence of the position angle could be explained if there are two superimposing components: one interstellar (constant) and the other - intrinsic (variable) with degree of polarization increasing toward the ultraviolet.

\section{Conclusions}

The simultaneous UBVRI photometry and polarimetry of naked $T$ Tau star HDE 283572 has enabled us to conclude that:

i) The distributions and the area of the active regions on the stellar surface are variable on time scale of a year.

ii) There is the intrinsic variable component of polarization with degree of polarization increasing toward the shorter wavelenght.

\section{References}

Walter, F.M.,Brown, A.,Linsky, J.L.,Rydgren, A.E.,Vrba, F., Roth, M., Carrasco, L., Chugainov, P.F., Shakhovskaya, N.I. and Imhoff C.L. (1987) 'X-ray sources in regions of star formation. II.The pre-main sequence G star HDE 283572', Astrophys.J., 314, 297-307. 